\title{
The Induction of Antioxidant Catalase Enzyme With Decrease of Plasma Malonidialdehyde: An Important Reactive Oxidative Species Inhibiting Mechanism
}

\author{
MAIJU RUOTTINEN ${ }^{1 *}$, VIIVI KAARONEN ${ }^{1 *}$, IINA SAIMANEN ${ }^{1 *}$, VIIVI KUOSMANEN ${ }^{1}$, \\ JARI KÄRKKÄINEN ${ }^{1}$, TUOMAS SELANDER ${ }^{2}$, SAMULI ASPINEN ${ }^{1}$ and MATTI ESKELINEN ${ }^{1}$ \\ ${ }^{1}$ Department of Surgery, Kuopio University Hospital and School of Medicine, \\ University of Eastern Finland, Kuopio, Finland; \\ ${ }^{2}$ Department of Science Service Center, Kuopio University Hospital, Kuopio, Finland
}

\begin{abstract}
Background/Aim: The simultaneous increase of antioxidant CAT (catalase) enzyme and plasma MDA (malonidialdehyde) concentrations versus the numeric rating scale (NRS) pain score following surgery is unknown. Patients and Methods: The study included 114 patients with gallstone disease and 29 patients in the cancer group. Results: Following surgery, the plasma CAT concentrations increased and plasma MDA concentrations decreased in all patients and especially in cancer patients. The linear mixed model time-effect was statistically significant in CAT and MDA $(p<0.001$ and $p=0.02$, respectively $)$. In addition, $a$ significant correlation between NRS pain score values and plasma MDA median concentrations in cancer patients was identified ( $r=0.430, p<0.001$ ). Conclusion: The plasma MDA concentrations decreased and CAT concentrations increased significantly in all patients and especially in cancer patients following surgery. The simultaneous increase of antioxidant CAT enzyme with the decrease of plasma MDA may be an important ROS inhibiting mechanism to help patients return to normal antioxidant-oxidant status.
\end{abstract}

Lipid peroxidation is a process where reactive oxygen species (ROS) attack lipids containing carbon double bonds (C-C),

This article is freely accessible online.

*These Authors contributed equally to this study.

Correspondence to: Matti Eskelinen, MD, School of Medicine, University of Eastern Finland, P.O. Box 100, FI-70029 KYS, Finland. Tel: +358 17173311, Fax: +358 17172611, e-mail: matti.eskelinen@kuh.fi

Key Words: Gallstone disease, cancer, surgery, plasma MDA, plasma CAT, NRS pain score. especially in polyunsaturated fatty acids (PUFAs). Lipid peroxidation of unsaturated lipids produces a variety of ROS products and has an important role in cell biology and human health. The main products of lipid peroxidation are lipid hydroperoxides (LOOH) while the secondary products consist of different aldehydes, of which the most harmful product is malonidialdehyde (MDA) (1-3). MDA is a toxic substance reacting with deoxyadenosine in DNA and forming DNA adducts, which are mutagenic and carcinogenic (1-3). The harmful effects of MDA could be neutralized by substances known as antioxidants such as vitamin $\mathrm{C}$ and vitamin $\mathrm{E}$, which may inhibit lipid peroxidation. Catalase (CAT) is one antioxidative substance, which plasma concentrations have shown to have inverse correlation to analgesic doses during the first 24 hours following surgery (4) and an inverse correlation to numeric rating scale (NRS) pain scores following surgery in midline laparotomy patients (5). Our previous article showed that the cancer patients with midline laparotomy had significantly lower plasma MDA concentrations postoperatively, whereas the postoperative alteration of median plasma CAT levels in cancer patients were significantly higher in the benign group of patients (6). Therefore, we felt it appropriate to carry out a study to compare the lipid peroxidation biomarker MDA levels to antioxidative biomarker CAT levels and NRS pain scores.

\section{Patients and Methods}

The study was approved by the Ethics Committee of Kuopio University Hospital District, Kuopio, Finland (DNRO 27/02/2013), it was registered in the ClinicalTrials.gov database (ClinicalTrials.gov Identifier: NCT01723540, Consort diagram, Figure 1), and was conducted in accordance with the Declaration of Helsinki. The study included 114 patients with cholelithiasis in the Laparoscopy Cholecystectomy (LC) (n=54) or Minilaparotomy Cholecystectomy (MC) $(n=60)$ groups (Figure 1) and the cancer group, recruited from our previous study, which included 10 patients with gastrointestinal cancer and 19 patients with gynecological cancer (7-9). The surgical 


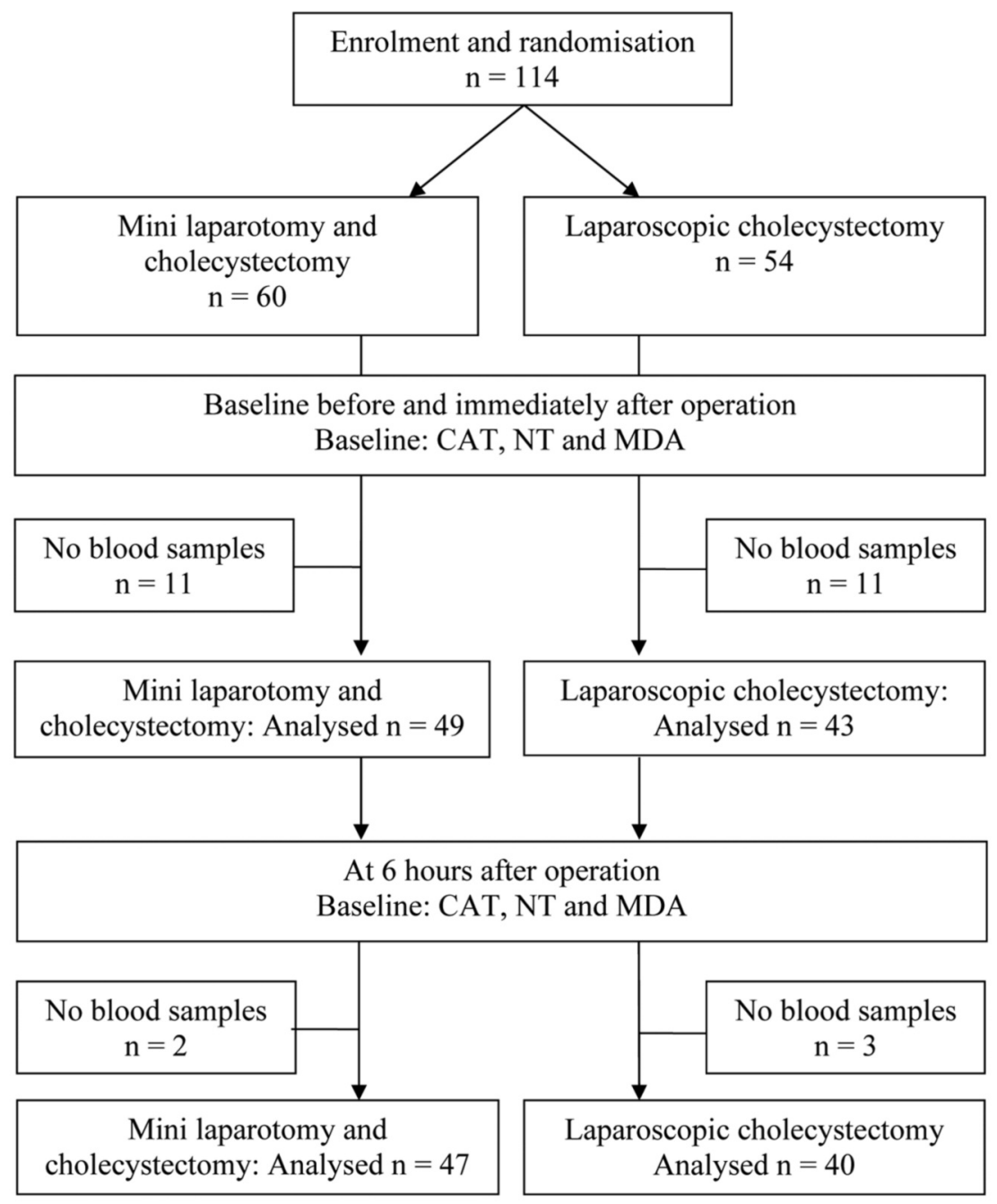

Figure 1. Study flow chart.

techniques used were standardized for both groups. The overall pain was measured using an 11-point numeric rating scale (NRS; $0=$ no pain; $10=$ most pain). The study protocol was fully described in our earlier original work (10-12).

EDTA-blood samples were taken before surgery (PRE), immediately after surgery (POP1) and $6 \mathrm{~h}$ post-operatively (POP2) and centrifuged at $1,000 \mathrm{G}(2,900 \mathrm{rpm})$ for $15 \mathrm{~min}$. The study protocol of CAT and NT were fully described in our earlier original works $(4,5,13,14)$. Plasma MDA concentrations were determined by using MDA (Malondialdehyde) ELISA Kit (E-EL-0060, Elabscience, Biotechnology Inc. Houston, Texas, USA). The manufacturer's intra-assay and the inter-assay coefficients of variation (CVs) were $5.6 \%$ and $6.28 \%$, respectively.

Data are presented as means and standard deviations or frequencies and percentages, where appropriate. Differences in baseline characteristics between groups were tested by the Fisher's exact test and in the case of continuous data, the analysis was performed by $t$-test. Group differences at three time points were tested by the Wilcoxon signed rank test and the Kruskall-Wallis-test.
Table I. Clinical data of the study groups. Data are mean (standard deviation) or number of cases.

\begin{tabular}{lccc}
\hline Variable & $\begin{array}{c}\text { Mini laparotomy } \\
\mathrm{n}=60\end{array}$ & $\begin{array}{c}\text { Laparoscopy } \\
\mathrm{n}=54\end{array}$ & $p$-Value \\
\hline Age, years & $50.7(13.2)$ & $53.2(13.1)$ & 0.316 \\
Gender male/female & $11 / 49$ & $18 / 36$ & 0.066 \\
Height, $\mathrm{cm}$ & $167.4(7.6)$ & $168.9(9.9)$ & 0.355 \\
Weight, $\mathrm{kg}$ & $77.3(14.4)$ & $83.0(17.4)$ & 0.057 \\
BMI, $\mathrm{kg} / \mathrm{m}^{2}$ & $27.6(4.4)$ & $29.1(5.6)$ & 0.111 \\
Operative time, min & $70.0(26.9)$ & $70.1(35.3)$ & 0.976 \\
Time in the operative & $120.4(28.5)$ & $127.1(35.8)$ & 0.287 \\
room, min & & & \\
Perioperative bleed, ml & $41(59)$ & $31(39)$ & 0.290 \\
Conversion rate, $\mathrm{n}$ & 3 & 3 & $1.000^{*}$ \\
Length of the skin & $49.7(12.1)$ & $78.3(22.7)$ & $<0.001$ \\
incision(s), mm & \multicolumn{3}{c}{} \\
\hline
\end{tabular}

BMI: Body mass index. $T$-test and *Fisher's exact test were used. 
Table II. Plasma catalase (CAT), nitrotyrosine (NT) and malonidialdehyde (MDA) concentrations in minicholecystectomy (MC) and laparoscopic cholecystectomy $(L C)$ patients versus cancer patients. Plasma concentrations were measured before (PRE), immediately after (POP1) and 6 h after (POP2) surgery. Median (interquartile range) concentrations are shown. Kruskall-Wallis test and linear mixed model was used.

\begin{tabular}{lcccc}
\hline Marker & MC & LC & Cancer & $p$-Value \\
\hline CAT (pg/ml) & & & & $<.001$ \\
$\quad$ PRE & $0.75(0.42-1.36)$ & $1.09(0.56-1.60)$ & $0.73(0.48-1.30)$ & 0.384 \\
POP1 & $0.79(0.48-1.37)$ & $0.93(0.56-1.65)$ & $1.46(1.13-2.36)$ & 0.001 \\
POP2 & $0.76(0.43-1.22)$ & $1.02(0.67-1.58)$ & $5.12(0.68-1.50)$ & 0.069 \\
NT (pg/ml) & & & $5.67(4.10-8.10)$ & $\mathbf{0 . 0 5 4}$ \\
PRE & $3.79(2.88-5.16)$ & $4.39(2.48-5.71)$ & $5.28(3.41-6.44)$ & 0.001 \\
POP1 & $4.11(2.96-5.26)$ & $4.40(2.76-5.76)$ & $4.69(4.09-6.27)$ & 0.078 \\
POP2 & $4.25(3.00-5.45)$ & & & 0.243 \\
MDA (ng/ml) & & $7.51(2.96-5.84)$ & $868(571-1037)$ & $<0.001$ \\
PRE & $877(646-1002)$ & $890(694-1186)$ & $589(392-730)$ & $<0.001$ \\
POP1 & $844(635-1093)$ & $905(606-1156)$ & $560(411-758)$ & $<0.001$ \\
POP2 & $864(682-1065)$ & & \\
\hline
\end{tabular}

Linear mixed effect model $p$-Values for interaction time group are in bold. Time-effect in linear mixed model in plasma catalase, nitrotyrosine and malonidialdehyde in cancer group was statistically significant $(p<0.001, p=0.009$ and $p<0.001$, respectively).

The plasma CAT, NT and MDA concentrations and differences in the patients with MC, LC and cancer were tested by the MannWhitney $U$-test. The linear mixed effect (LME) model was used to test the interaction group time effect. In LME analysis plasma concentrations were $\log$ transformed. The results of the laboratory measurements are presented as median concentrations with interquartile range as distributions were skewed to the right. The Pearson's method was used to test for correlation for plasma NT concentrations versus MDA concentrations. Data were analyzed by IBM SPSS statistical software (IBM Corp. released 2013, IBM SPSS Statistics for Windows, version 22.0, IBM Corporation, Armonk, NY, USA). $p$-Values under 0.05 were considered statistically significant.

\section{Results}

In cholecystectomy patients, randomized either in the MC or LC groups, there were no significant difference in mean age, height, weight, mean body mass index (BMI), operative time, time in the operative room, perioperative bleed or conversion rate. The skin incision was significantly longer in the LC than in the MC group $(p<0.001$, Table I).

The median plasma oxidative stress concentrations of the NT and MDA biomarkers between the MC and LC groups were quite similar (Table II). Changes in plasma MDA and NT concentrations following surgery are shown in Table II. Lipid peroxidation biomarker MDA increased significantly following surgery in LC patients and decreased significantly in cancer patients (Table II). The statistically significant linear mixed model (LME) $p$-Values for interaction time in MC patients, LC patients and cancer patients are shown in Table II $(p<0.001, p=0.05$ and $p<0.001$, respectively). In cancer patients, the LME time-effect was statistically significant in CAT, NT and MDA concentrations $(p<0.001$,
Table III. Alteration of plasma catalase (CAT), nitrotyrosine (NT) and malonidialdehyde (MDA) concentrations following surgery. Plasma concentrations were measured before (PRE), immediately after (POP1) and 6 h after operation (POP2). Median (interquartile range) concentrations are shown.

\begin{tabular}{lccc}
\hline Marker & All patients & Alteration & $p$-Value \\
\hline CAT $(\mathrm{pg} / \mathrm{ml})$ & & & $\mathbf{0 . 0 0 1}$ \\
PRE & $0.79(0.44-1.50)$ & PRE $v s$. POP1 & 0.001 \\
POP1 & $1.01(0.56-1.68)$ & POP1 vs. POP2 & 0.027 \\
POP2 & $0.92(0.57-1.48)$ & & \\
NT $(\mathrm{pg} / \mathrm{ml})$ & & & $\mathbf{0 . 0 7 1}$ \\
PRE & $4.38(3.40-5.58)$ & PRE $v s$. POP1 & 0.039 \\
POP1 & $4.16(3.17-5.37)$ & POP1 vs. POP2 & 0.077 \\
POP2 & $4.31(3.38-5.51)$ & & \\
MDA $(\mathrm{ng} / \mathrm{ml})$ & & & $\mathbf{0 . 0 2 0}$ \\
PRE & $880(649-1128)$ & PRE $v s$. POP1 & 0.001 \\
POP1 & $839(589-1127)$ & POP1 vs. POP2 & 0.943 \\
POP2 & $857(600-1132)$ & & \\
\hline
\end{tabular}

The Wilcoxon signed-rank test and linear mixed model was used. Linear mixed model $p$-Values for time effect are in bold.

$p=0.009$ and $p<0.001$, respectively). The antioxidant plasma CAT concentrations increased and plasma MDA decreased significantly in all patients and especially in cancer patients (Table II). The LME time-effect of the plasma CAT and MDA concentrations preoperatively and following surgery was significant ( $p=0.001$ and $p=0.02$, respectively, Table III).

There was a significant correlation in the NRS pain score values versus the plasma MDA values in cancer patients $(r=0.430, p<0.001$, Figure 2$)$ and a significant correlation in the median MDA versus median NT plasma concentrations in all patients was found ( $r=0.445, p<0.001$, Figure 3$)$. 


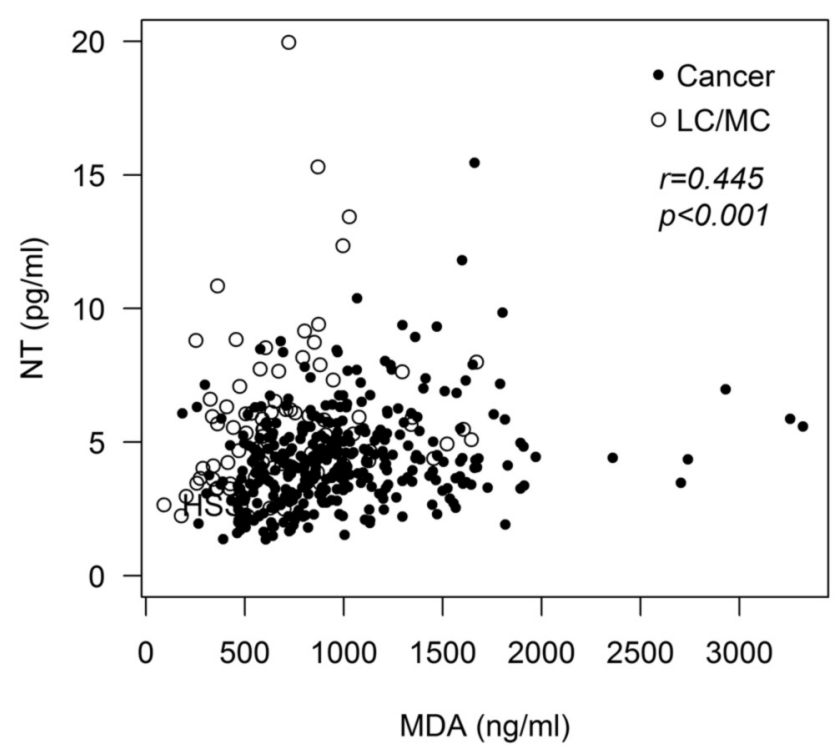

Figure 2. Scatter plots of the plasma malonidialdehyde (MDA) concentration versus pain assessed using a 11-point numeric rating scale (NRS; $0=$ no pain; $10=$ most pain) at 8 hours postoperatively $\left(N R S_{8}\right)$ in cancer patients $(r=0.430, p<0.001)$.

\section{Discussion}

MDA is an end product of lipid peroxidation of PUFAs located in cell membranes forming DNA adducts, which are mutagenic (1-3). Earlier studies of MDA biomarker are showing the connection between oxidative stress and cancer $(2,3)$, cardiovascular diseases $(2,3)$, diabetes $(2,3,16)$, liver disease (2, 3), Alzheimer's disease (1, 2, 3, 17, 18), Parkinson's disease (1) and major depressive disorders (19). In addition, MDA is used as a biomarker of acetaminophen hepatotoxicity (20) and to identify ROS changes of bladder tissue in the initial phase of diabetes (16). Tsounapi et al. (16) showed that MDA levels in the bladder wall were significantly higher in the diabetes group compared to other study groups. They also suggested that antioxidant substances such as taurine and resveratrol could provide cytoprotection of bladder tissue by inhibiting MDA (16).

Lipid peroxidation, ROS and reactive nitrogen species (RNS) results in an imbalance between the formation of ROS species and decreased ability to detoxify and to inhibit the damage. The negative effects of ROS/RNS can be neutralized by substances known as antioxidants. Living cells contain large number of antioxidants, vitamin $\mathrm{C}$ and $\mathrm{E}$, carotenoids and flavonoids $(21,22)$. Interestingly, oligomeric proanthocyanidins (OPCs) extracted from grape seeds have been shown to have higher antioxidant capability than vitamin C or E (21). Lu et al. (24) studied the effect of OPCs and showed that OPC supplementation significantly reduced the plasma MDA biomarker concentrations and increased the

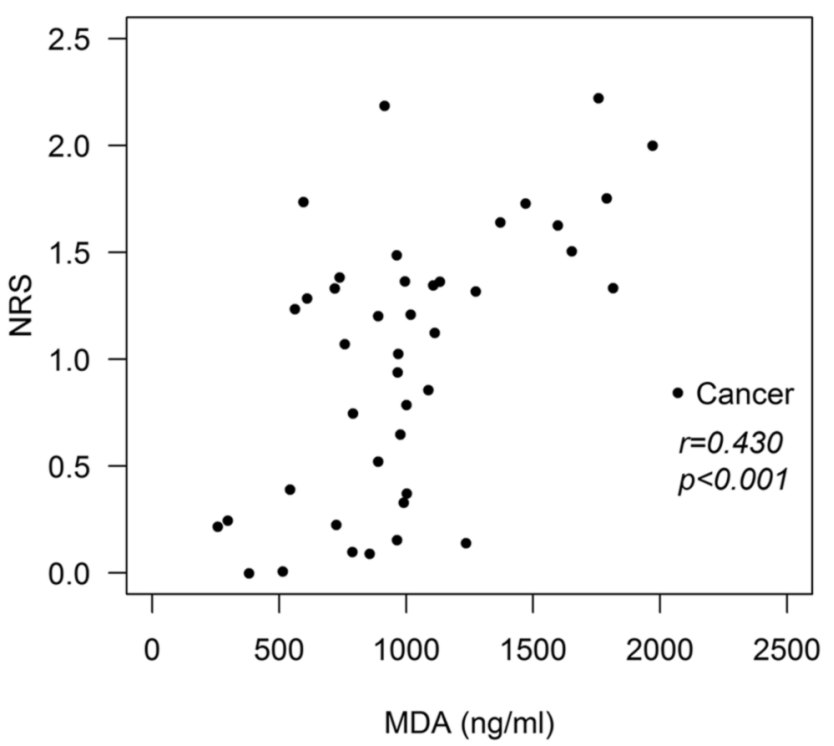

Figure 3. Jitterplot of plasma malonidialdehyde (MDA) concentration versus plasma nitrotyrosine $(N T)$ concentration in cholecystectomy and cancer patients $(r=0.445, p<0.001)$.

plasma CAT concentrations, thereby inhibiting the ROS substances and increasing the antioxidant capacity of patients with COPD (Chronic Obstructive Pulmonary Disease).

The most common method used to measure MDA in the earlier studies is the thiobarbituric acid substances (TBARS) assay. The TBARS test is based on the reactivity of thiobarbituric acid toward MDA. This test was first used by food chemists to evaluate degradation of fats and oils (3). Although, TBARS is not specific enough due to interference from substances that are produced during the assay and presence of false chromogens, Spanidis et al. (23) showed that TBARS reactive substances detection is a useful biomarker of oxidative stress in obese sepsis patients in the intensive care. Several methods for measuring free and total MDA are available; including gas chromatography mass spectrometry and liquid chromatography-mass spectrometry (8). In addition, the ELISA sandwich assay has also been used to measure plasma MDA concentrations (6).

The median plasma concentrations of NT and MDA between the MC and LC patients were quite similar and it seems that cholecystectomy, in either MC or LC, causes only a moderate oxidative stress response. However, the median plasma MDA concentrations decreased and CAT concentrations increased significantly in all patients and especially in cancer patients following surgery. In addition, the decrease of plasma MDA and increase in plasma CAT concentrations showed statistically significant $p$-Values in the linear mixed model. Accordingly, the simultaneous increase of the antioxidant CAT enzyme with the decrease of 
lipid peroxidation biomarker MDA may be an important ROS inhibiting mechanism to help patients return normal antioxidant-oxidant status. In addition, our results of a significant correlation between the plasma MDA levels versus the NRS pain scores are in line with the results shown in the systematic review of Yiannakopoulou et al. (26).

In conclusion, the efficient inhibition of ROS requires coordinate action of several cellular antioxidant enzymes. In this study, the median plasma CAT concentrations in all patients and especially in cancer patients increased significantly following surgery and the antioxidant CAT enzyme could help patients return normal antioxidantoxidant status via enhancing CAT to neutralize ROS substances and to supress oxidative stress.

\section{Conflicts of Interest}

The Authors report no conflicts of interest or financial ties to disclose. The Authors alone are responsible for the content and writing of this article.

\section{Authors' Contributions}

MR, VK, IS, VK, JK, SA and ME were responsible for the recruitment of study participants and clinical and laboratory investigations and collection of data. TS performed the statistical analyses. All authors contributed to the data analysis, drafting and revising the manuscript, read and approved the final manuscript.

\section{Acknowledgements}

The study was funded by the Päivikki ja Sakari Sohlberg Foundation.

\section{References}

1 Akyol O, Zoroglu SS, Armutcu F, Sahin S and Gurel A: Nitric oxide as a physiopathological factor in neuropsychiatric disorders. In Vivo 18: 377-390, 2004. PMID: 15341194.

2 Del Rio D, Stewart AJ and Pellegrini N: A review of recent studies on malondialdehyde as toxic molecule and biological marker of oxidative stress. Nutr Metab Cardiovasc Dis 15: 316328, 2005. PMID: 16054557. DOI: 10.1016/j.numecd.2005. 05.003

3 Ayala A, Muñoz MF and Argüelles S: Lipid peroxidation: Production, metabolism, and signaling mechanisms of malondialdehyde and 4-hydroxy-2-nonenal. Oxid Med Cell Longev 2014: 360438, 2014. PMID: 24999379. DOI: 10.1155/ 2014/360438

4 Saimanen I, Kuosmanen V, Kärkkäinen J, Selander T, Aspinen S, Holopainen A, Rantanen T and Eskelinen M: Cholecystectomy patients with high plasma level of catalase have significantly lower analgesia requirement: A prospective study of two different cholecystectomy techniques with special reference to patients with cancer. Anticancer Res 38: 5417-5422, 2018. PMID: 30194197. DOI: 10.21873 /anticanres.12872

5 Saimanen I, Kärkkäinen J, Selander T, Purdy M, Kokki M, Kokki $\mathrm{H}$ and Eskelinen M: Plasma catalase in relation to pain following midline laparotomy: A prospective study of patients with benign diseases and patients with cancer. Anticancer Res 38: 6479-6484, 2018. PMID: 30396975. DOI: 10.21873/ anticanres. 13011

6 Ruottinen M, Kuosmanen V, Saimanen I, Kaaronen V, Rahkola D, Holopainen A, Selander T, Kokki H, Kokki M and Eskelinen M: The rectus sheath block (RSB) analgesia following laparotomy could affect malonidialdehyde (MDA) concentrations in benign disease and cancer. Anticancer Res 40: 253-259, 2020. PMID: 31892574. DOI: 10.21873/anticanres.13947

7 Purdy M, Kokki M, Anttila M, Aspinen S, Juvonen P, Selander T, Kokki H, Pulkki K and Eskelinen M: Does the post-surgery placement of rectus sheath block analgesia alter the oxidative stress biomarker 8-OHdG concentrations: A randomised trial of patients with cancer and benign disease. Cancer Genomics \& Proteomics 13: 239-244, 2016. PMID: 27107066.

8 Purdy M, Kokki M, Anttila M, Aspinen S, Juvonen P, Korhonen $\mathrm{R}$, Selander T, Kokki H and Eskelinen M: Does the rectus sheath block analgesia reduce the inflammatory response biomarker IL1ra, IL-6, IL-8, IL-10 and IL-1 $\beta$ concentrations following surgery? A randomised clinical trial of patients with cancer and benign disease. Anticancer Res 36: 3005-3011, 2016. PMID: 27272818. DOI: 10.21873 /anticanres.11396

9 Purdy M, Kärkkäinen J, Kokki M, Anttila M, Aspinen S, Juvonen P, Kokki H, Pulkki K, Rantanen T and Eskelinen M: Does rectus sheath block analgesia alter levels of the oxidative stress biomarker glutathione peroxidase: A randomised trial of patients with cancer and benign disease. Anticancer Res 37: 897902, 2017. PMID: 28179349. DOI: 10.21873/anticanres.11396

10 Kärkkäinen J, Aspinen S, Harju J, Juvonen P, Pulkki K and Eskelinen M: Plasma glutathione peroxidase (GPX1) levels and oxidative stress in gallstone patients operated with two different cholecystectomy techniques: A randomized study with special reference to cancer patients. Anticancer Res 37: 6921-6927, 2017. PMID: 29187474. DOI: 10.21873/anticanres.12156

11 Kärkkäinen J, Selander T, Purdy M, Juvonen P and Eskelinen M: Patients with increased levels of the oxidative stress biomarker SOD1 appear to have dimished postoperative pain after midline laparotomy: A randomized trial with special reference to postoperative pain score (NRS). Anticancer Res 38: 1003-1008, 2018. PMID: 29374733. DOI: 10.21873/anticanres. 12315

12 Kärkkäinen J, Saimanen I, Selander T, Aspinen S, Harju J, Juvonen P and Eskelinen M: Gallstone patients with enhanced oxidative stress biomarker SOD1 plasma levels have significantly lower number of postoperative analgesic oxycodone doses: A prospective study with special reference to cancer patients. Anticancer Res 38: 3573-3578, 2018. PMID: 29848712. DOI: 10.21873 /anticanres.12630

13 Saimanen I, Rahkola D, Kuosmanen V, Kärkkäinen J, Selander T, Holopainen A, Aspinen S and Eskelinen M: Nitrotyrosine (NT), a nitrosative stress biomarker, plasma concentrations in gallstone disease and cancer patients. Anticancer Res 39: 809814, 2019. PMID: 30711961. DOI: 10.21873/anticanres.13179

14 Kuosmanen V, Saimanen I, Rahkola D, Kärkkäinen J, Selander T, Purdy M, Kokki H, Kokki M and Eskelinen M: Rectus sheath block (RSB) analgesia could enhance significantly the patient satisfaction following midline laparotomy in benign disease and in cancer: A prospective study with special reference to nitrosative stress marker nitrotyrosine (NT) plasma concentrations. 
Anticancer Res 39: 1383-1389, 2019. PMID: 30842172. DOI: 10.21873/anticanres. 13252

15 Dogaru G, Bulboaca A, Boarescu PM, Ciumarnean L, Rus V, Sitar-Taut AV, Munteanu C, Bodisz G and Stanescu I: The effect of mofettes on oxidative stress/antioxidant balance in experimental myocardial ischemia. In Vivo 33: 1911-1920, 2019. PMID: 31662519. DOI: 10.21873/invivo.11685

16 Tsounapi P, Honda M, Hikita K, Sofikitis N and Takenaka A: Oxidative stress alterations in the bladder of a short-period type 2 diabetes rat model: Antioxidant treatment can be beneficial for the bladder. In Vivo 33: 1819-1826, 2019. PMID: 31662508. DOI: 10.21873 /invivo. 11674

17 Calabrese V, Boyd-Kimball D, Scapagnini G and Butterfield DA: Nitric oxide and cellular stress response in brain aging and neurodegenerative disorders: the role of vitagenes. In Vivo 18: 245-267, 2004. PMID: 15341181.

18 Seyidova D, Aliyev A, Rzayev N, Obrenovich M, Lamb BT, Smith MA, de la Torre JC, Perry G and Aliev G: The role of nitric oxide in the pathogenesis of brain lesions during the development of Alzheimer's disease. In Vivo 18: 325-333, 2004. PMID: 15341188.

19 Mazereeuw G, Herrmann N, Andreazza AC, Khan MM and Lanctôt KL: A meta-analysis of lipid peroxidation markers in major depression. Neuropsychiatric Dis Treat 11: 2479-2491, 2015. PMID: 26491326. DOI: 10.2147/NDT.S89922

20 Dogaru G, Bulboaca AE, Gheban D, Boarescu PM, Rus V, Festila D, Sitar-Taut A-V and Stanescu I: Effect of liposomal curcumin on acetaminophen hepatotoxicity by down-regulation of oxidative stress and matrix metalloproteinases. In Vivo 34: 569-582, 2020. PMID: 32111755. DOI: 10.21873/invivo.11809

21 Carini F, Mazzola M, Rappa F, Jurjus A, Geagea AG , Al Kattar S, Bou-Assi T, Jurjus R, Damiani P, Leone A and Tomasello G: Colorectal carcinogenesis: Role of oxidative stress and antioxidants. Anticancer Res 37: 4759-4766, 2017. PMID: 28870894. DOI: 10.21873 /anticanres.11882
22 Mokbel K, and Mokbel K: Chemoprevention of breast cancer with vitamins and micronutrients: A concise review. In Vivo 33: 983-997, 2019. PMID: 31280187. DOI: 10.21873/invivo.11568

23 Uchiyama H, Uehara K, Nagashima T, Nakata A, Sato K, Mihara Y, Komatsu KI, Takanari J, Shimizu S and Wakame K: Global liver gene expression analysis on a murine metabolic syndrome model treated by low-molecular-weight lychee fruit polyphenol (Oligonol ${ }^{\circledR}$ ). Anticancer Res 36: 3705-3713, 2016. PMID: 27354644.

24 Lu MC, Yang MD, Li PC, Fang HY, Huang HY, Chan YC and Bau DT: Effect of oligomeric proanthocyanidin on the antioxidant status and lung function of patients with chronic obstructive pulmonary disease. In Vivo 32: 753-758, 2018. PMID: 29936455. DOI: 10.21873/invivo.11304

25 Spanidis Y, Goutzourelas N, Stagos D, Kolyva AS, Gogos CA, Bar-Or D and Kouretas D: Assessment of oxidative stress in septic and obese patients using markers of oxidation-reduction potential. In Vivo 29: 595-600, 2015. PMID: 26359419.

26 Yiannakopoulou ECh, Nikiteas N, Perrea D and Tsigris C: Effect of laparoscopic surgery on oxidative stress response: systematic review. Surg Laparosc Endosc Percutan Tech 23: 101-108, 2013. PMID: 23579502. DOI: 10.1097/SLE.0b013e3182827b33

Received June 15, 2020

Revised July 17, 2020

Accepted July 22, 2020 